\title{
Alterität und Heroismus in Franz Grillparzers Trilogie Das Goldene Vließ und in Euripides' Medeia
}

\author{
Mario Zanucchi
}

\begin{abstract}
Alterität und Heroismus bilden zwei herausragende Themenkomplexe in Grillparzers großem dramatischem Wurf Das Goldene Vließ. ${ }^{1}$ Nach einer Einleitung zu Ent-
\end{abstract}

1 Die Trilogie wird im Folgenden nach der Ausgabe von Helmut Bachmaier zitiert (Franz Grillparzer: Werke in sechs Bänden, hg. von Helmut Bachmaier, Bd. 2: Dramen 1817-1828. Frankfurt am Main 1986). Die Forschungsliteratur zu Grillparzers Trilogie ist umfangreich, wiewohl noch keine Spezialforschung zur Problematik des Heroischen vorliegt. $\mathrm{Zu}$ den bedeutendsten Untersuchungen zählen folgende Studien: Rudolf Stiefel: Grillparzers, Goldenes Vließ'. Ein dichterisches Bekenntnis (Basler Studien zur deutschen Sprache und Literatur; 21). Bern 1959. Ulrich Fülleborn: Zu Grillparzers Goldenem Vließ. Der Sinn der Raum- und Zeitgestaltung. In: Jahrbuch der Grillparzer-Gesellschaft 12, 1976, 39-59. Konrad Kenkel: Medeadramen. Entmythisierung und Remythisierung. Euripides, Klinger, Grillparzer, Jabnn, Anouilh (Studien zur Germanistik, Anglistik und Komparatistik; 63). Bonn 1979. Jean-Louise Bandet: Corneille - Grillparzer - Anouilh. Zur Behandlung des Medea-Stoffes in Österreich und Frankreich. In: Robert Pichl [et al.] (Hg.): Grillparzer und die europäische Tradition. Wien 1987, 31-43. Matthias Brauckmann und Andrea Everwien: Sehnsucht nach Integrität oder Wie die Seele wächst im Verzicht: Das goldene Vließ. In: Bernhard Budde und Ulrich Schmidt (Hg.): Gerettete Ordnung. Grillparzers Dramen (Historisch-kritische Arbeiten zur deutschen Literatur; 7). Frankfurt am Main [u.a.] 1987, 58-105 und 320-329. Rolf Geißler: Grillparzers Goldenes Vließ und Goethes Faust als Kritik neuzeitlicher Geschichte. In: Jabrbuch der GrillparzerGesellschaft 18, 1991/92, 257-270. Hilde Haider-Pregler: Grillparzers Trilogie Das goldene Vließ. Dramaturgie und Rezeption. In: Helmut Bachmaier (Hg.): Franz Grillparzer. Frankfurt am Main 1991, 273-320. Hans-Georg Werner: Verteufelt human. Etwas über den Zusammenhang zwischen Goethes Iphigenie und Grillparzers Goldenem Vließ. In: Hans-Georg Werner: Literarische Strategien. Studien zur deutschen Literatur 1760 bis 1840. Stuttgart und Weimar 1993, 229-242. Wolfgang F. Bender: „Das Drama lügt eine Gegenwart.“ Versinnlichung in Franz Grillparzers Trilogie Das goldene Vließ. In: Hilde Haider-Pregler und Evelyn Deutsch-Schreiner (Hg.): „Stichwort Grillparzer“(Grillparzer-Forum; 1). Wien [u.a.] 1994, 97106. Gerhard Neumann: Das goldene Vließ. Die Erneuerung der Tragödie durch Grillparzer. In: Hellmut Flashar (Hg.): Tragödie. Idee und Transformation (Colloquium Rauricum; 5). Stuttgart und Leipzig 1997, 258-286. Paola Gheri: Franz Grillparzer. Mito e critica della cultura in Das goldene Vließ. In: Gabriella Catalano und Emilia Fiandra (Hg.): Ottocento tedesco. Da Goethe a Nietzsche. Neapel 1998, 255-278. Tillmann Bub: Barbarei und Zivilisation in Grillparzers Trilogie Das goldene Vließ. In: Sprachkunst 35: 1, 2004, 1-22. Jeanine CharueFerrucci: La notion de Barbarie dans la trilogie de Grillparzer La Toison d'Or (Das Goldene Vließ). In: Pierre Labaye (Hg.): L'Allemagne, des Lumières à la Modernité. Mélanges offerts à JeanLouis Bandet (Études germaniques). Rennes 1997, 133-143. Karina Becker: Autonomie und Humanität. Grenzen der Aufklärung in Goethes „Iphigenie“", Kleists „Penthesilea“ und Grillparzers „Medea“ (Historisch-kritische Arbeiten zur deutschen Literatur; 44). Frankfurt am Main [u.a.] 2008. Friederike Raphaela Lanz: „Weil eine Fremd” ich bin, aus fernem Land ... “. Fremdheit und Fremde im dramatischen Werk Franz Grillparzers und Friedrich Hebbels. Mainz, Diss. 2009. Markus Winkler: Von Iphigenie zu Medea. Semantik und Dramaturgie des Barbarischen bei Goethe und Grillparzer (Untersuchungen zur deutschen Literaturgeschichte; 133). Tübingen 2009. 
stehung, Quellen und Aufbau der Trilogie soll zunächst die Problematik der kulturellen Alterität angesprochen werden, wie sie sich zum einen in Grillparzers Konstruktion von Kolchis niederschlägt, zum anderen im culture clash zeigt, der sich aus Medeas Übersiedlung von Kolchis nach Korinth ergibt. Sodann soll die Heroismusproblematik ins Visier genommen und an der Jason-Figur Grillparzers Depotenzierung der maskulinen und westlichen Heldentumsrhetorik rekonstruiert werden. Den Beitrag schließt ein kontrastiver Vergleich des Goldnen Vließes mit Euripides' Medeia ab, der dazu dienen soll, die Spezifik von Grillparzers Medea schärfer herauszuarbeiten.

Franz Grillparzers Trilogie Das Goldene Vlie $\beta$ - „ein dramatisches Gedicht in drei Abteilungen", so der Untertitel - entstand zwischen 1818 und 1821. Den Hinweis auf den Medea-Stoff erhielt Grillparzer dank August Wilhelm Schlegels Vorlesungen Über dramatische Kunst und Literatur (1809), die auch eine kritische Würdigung von Euripides' Medeia enthalten. ${ }^{2}$ Die Tragödie des Euripides, den Grillparzer später in seinen Studien immer wieder gegenüber Schlegel verteidigte, bildet die Hauptquelle. ${ }^{3}$ Von Bedeutung war auch Grillparzers Lektüre von Senecas Tragödie, aus der er Medeas Auftrittsmonolog übersetzte, ${ }^{4}$ sowie weiterer antiker Bearbeitungen des Mythos wie die Argonautica des Apollonius Rhodius. Anregungen verdankt er überdies Calderóns mythologischer Tragödie Los tres mayores prodigios (1636), dessen I. Akt den Medea-Stoff dramatisiert. Es war aber seiner Selbstbiographie zufolge der Medea-Artikel in Benjamin Hederichs Mythologischem Lexikon ${ }^{5}$ mit seiner Schilderung Medeas als einer der größten Zauberinnen, der ihn dazu verleitete, den Unterschied zwischen Kolchis und Griechenland in den Vordergrund zu rücken. Deswegen gestaltete Grillparzer auch den überlieferten Tragödienstoff zu einer Trilogie um.

De facto handelt es sich bei Grillparzers Goldenem Vließ allerdings um ein Doppeldrama, da der erste und zweite Teil zusammen als Fünfakter gelten könnten. ${ }^{6}$ Die triadische Einteilung mag eine Schiller-Ämulation darstellen, dessen „dramatisches Gedicht“ Wallenstein (1800) ebenfalls als Trilogie angelegt ist. Grillparzer legte größten Wert darauf, die drei Abteilungen der Trilogie - Der Gastfreund, Die Argonauten

2 August Wilhelm Schlegel: Kritische Schriften und Briefe, hg. von Edgar Lohner, Bd. 5: Vorlesungen über dramatische Kunst und Literatur. Erster Teil. Stuttgart [u.a.] 1966, 122.

3 Zu Grillparzers Euripides-Rezeption vgl. Wendelin Schmidt-Dengler: Grillparzer liest Euripides. Notizen zu einem ungewöhnlichen Lektürevorgang. In: Jabrbuch der GrillparzerGesellschaft 3, 1991/92, 329-339.

4 Franz Grillparzer: Sämtliche Werke. Ausgewählte Briefe, Gespräche, Berichte, hg. von Peter Frank und Karl Pörnbacher, Bd. 2: Dramen 2. Jugenddramen. Dramatische Fragmente und Pläne. München 1961, 1219-1220.

5 Grillparzer konsultierte Hederichs Lexikon in der 1770 von Joachim Schwabe besorgten Ausgabe.

6 Friedrich Sengle: Biedermeierzeit. Deutsche Literatur im Spannungsfeld zwischen Restauration und Revolution 1815-1848, Bd. 3: Die Dichter. Stuttgart 1980, 86. 
und Medea - unbedingt zusammen an zwei aufeinanderfolgenden Tagen aufzuführen. Er bat auch die Hoftheaterdirektion in einem Brief, die drei Abteilungen an zwei Tagen zu geben, da "diese sich wechselseitig bedingen und erklären".7 Schon zu Grillparzers Lebzeiten wurde allerdings nur der dritte Teil aufgeführt.

Dadurch wurde die Tragödie gerade ihrer innovativen Aspekte beraubt, die vor allem in den beiden ersten, der Vorgeschichte Medeas geltenden, Teilen der Trilogie zur Geltung kommen. ${ }^{8}$ Als erster Dramenautor bringt Grillparzer nämlich die Vorgeschichte, die sich in Kolchis abspielt, auf die Bühne. Dadurch schwächt er den überlieferungsgeschichtlich relevantesten Aspekt des Medea-Stoffes, den Kindermord, rückt stattdessen das kolonialistische Streben nach dem Besitz des Vlieses in den Vordergrund und verleiht auch der exotischen Heldin Medea schärfere Konturen. Darin folgt er weniger Euripides, der Medeia gräzisiert," ${ }^{9}$ als vielmehr dem von ihm übersetzten Seneca, welcher der Heldin deutlich barbarischere Züge verleiht. Symptomatisch dafür ist in Senecas Tragödie die große Beschwörungsszene im IV. Akt, in dem Medea in Trance Gewand und Geschmeide für Kreusa verzaubert und zugleich vorolympische Gottheiten evoziert, unter anderem den Giganten Typhoeus, der gegen Zeus rebelliert hatte. Seneca aber ging über den überlieferten Tragödienstoff nicht hinaus. Es war dagegen im 19. Jahrhundert Grillparzer, der als erster der Vorgeschichte Medeas ein dramatisches Eigengewicht

7 Grillparzer: Werke in sechs Bänden, Bd. 2 (Anm. 1), 790 (Brief an die Hoftheaterdirektion vom 8. November 1820, Entwurf).

8 Dies betont auch Ulrich Fülleborn, der diese „kühne Innovation“ als Ausgangspunkt zu seiner Interpretation der Trilogie nahm (Fülleborn: Zu Grillparzers Goldenem Vließ (Anm. 1)).

9 Euripides' Medeia erscheint zwar als eine Wilde; keine griechische Frau hätte ihre Untat über

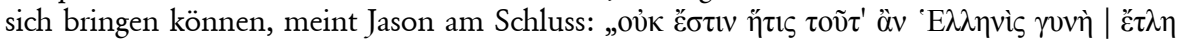
$\pi \mathrm{o} \theta^{\prime \prime \prime}$ (Euripides: Medea, hg. von Donald J. Mastronarde. Cambridge 2002, Z. 1339-1340). Sie huldigt aber denselben Göttern wie die Griechen, die offenbar auch ihre Rache an Jason begünstigen: vor allem Zeus, Themis und Artemis. Lediglich Medeias Anrufung des Sonnengottes Helios, der allerdings auch in Rhodos kultisch verehrt wurde, sowie vor allem der

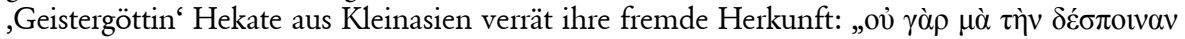

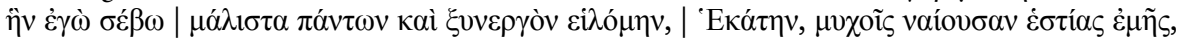

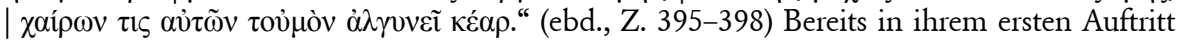
auf der Bühne identifiziert sie sich mit ihrer neuen Existenz einer athenischen Ehefrau, und auch die Begründung ihres Racheplans beruht auf griechisch-heroischen Werten (Georg Otten: Die Medea des Euripides. Ein Kommentar zur deutschen Übersetzung. Berlin 2005, 356-357). Bezeichnend für Euripides' Hellenisierung Medeias ist auch, dass das Wort $\beta \alpha ́ \rho \beta \alpha \rho o \varsigma$ in seiner Tragödie spärlich vorkommt. Die Vokabel ist insgesamt nur vier Mal belegt (Z. 256, 591, 536, 1330). Vor Euripides war Medeia bereits vom korinthischen Dichter Eumelos gräzisiert worden. Dessen Epos Korinthiaka schreibt Aietes eine korinthische Abstammung zu und lässt seine Tochter Medeia per Deszendenzrecht gar zur Königin von Korinth werden: „Eumelos sagte, dass Helios das Land am Asopos Aloeus gab und dass er dem Aietes Ephura zuwies. Als Aietes nach Kolchis aufbrach, übergab er sein Land Bunus, dem Sohn von Hermes und Alkidamea, und als Bunus starb, dehnte Epopeus, der Sohn von Aloeus, sein Königreich aus, indem er Ephura eingliederte. Als danach Korinthus, der Sohn von Marathon, kinderlos starb, holten die Korinther Medeia aus Iolkos und übergaben ihr das Königreich. Durch sie wurde Jason Herrscher von Korinth" (Pausanias: Graeciae descriptio 2.3.10). 
verlieh und somit die Antithese der Gegenwelten Kolchis und Griechenland ins Zentrum rückte.

\section{Grillparzers Kolchis}

In der ältesten mythischen Überlieferung ist Medeas Herkunftsland geografisch noch unbestimmt. Es handelt sich um das unbekannte, mythisch ferne Land Aia, das am Rande des Okeanos liegt und in dem der Gott Helios seinen Tageslauf beginnt. ${ }^{10}$ Nachdem später die fernen Gestade des Pontos bekannt geworden waren, identifizierte man das sagenhafte Aia mit Kolchis, das für die Griechen einen besonders exotischen Landstrich darstellte. ${ }^{11}$ Die in Kolchis ansässigen Kartweler unterschieden sich nämlich so stark in Sprache und Kultur von den anderen indoeuropäischen Stämmen, dass man diese Unterschiede durch Migrationshypothesen zu erklären versuchte. So argumentiert Herodot, die Kolcher seien ägyptischer Herkunft, Soldaten des sagenhaften ägyptischen Herrschers Sesostris, der sie am Phasis zurückließ. Er könne dies aus eigener Anschauung bestätigen, da die Kolcher eine schwarze Hautfarbe und krauses Haar hätten und die Männer beschneiden würden. Weiterhin würden Ägypter und Kolcher sich in der Leinenherstellung, überhaupt in Lebensweise und Sprache ähneln:

Offenbar sind die Bewohner von Kolchis Ägypter; ich habe das bemerkt, bevor man mir darüber von fremder Seite Auskunft gab. Als mir dieser Gedanke einfiel, fragte ich bei beiden Völkern nach. Die Kolcher erinnerten sich lebhafter an die Ägypter als die Ägypter an die Kolcher. Doch sagten mir die Ägypter, die Kolcher rührten nach ihrer Meinung noch vom Heer des Sesostris her. Ich selbst vermutete das auch, weil sie dunkelfarbig und kraushaarig sind. Das will freilich noch nicht viel bedeuten; denn andere Völker sind das auch. Aber aus folgendem ergibt es sich noch deutlicher: Kolcher, Aithioper und Ägypter beschneiden sich als einzige von allen Menschen die Geschlechtsteile von jeher. [...] Ich will noch etwas anderes erzählen, worin die Kolcher den Ägyptern ähnlich sind. Sie allein fertigen in gleicher Weise wie die Ägypter Leinwand an; ihre ganze Lebensweise und Sprache ist einander ähnlich. ${ }^{12}$

$\mathrm{Ob}$ allerdings die Kolcher damals wirklich dunkelhäutig waren, ist ebenso unsicher wie Herodots Reise ans Schwarze Meer. ${ }^{13}$ Die Dunkelhäutigkeit der Kolcher hätte Herodot nämlich auch Pindars vierter Pythischer Ode (im Jahr 462 v. Chr. aufgeführt) entnehmen können:

10 Mimnermos fr. 11. 12 Bgk. Vgl. Jakob Escher: Art. „Aia“. In: Paulys Realencyclopädie der classischen Altertumswissenschaft, Bd. I/1. Stuttgart 1893, Sp. 919-920.

11 Eumelos fr. 2. 8 (G. Kinkel (Hg.): Epicorum Graecorum fragmenta, Bd. 1. Leipzig 1877) sowie Herodot I 2. VIII 193. 197.

12 Herodot II 104 (Übersetzung nach: Herodot: Historien, Bd. 1, hg. und übers. von Josef Feix. München 1963, 285).

13 Die den Kolchern nachgesagte Dunkelhäutigkeit wird in der Antike nicht von allen Kennern der Länder an den Schwarzmeerküsten bestätigt. In der hippokratischen Schrift Über Winde, Wasser und Ortslagen werden die Anwohner des Phasis sogar als mit bleicher Haut wie bei den Gelbsüchtigen geschildert (Hippokrates: Peri Aëron, Hydaton, Topon, XV 20-24). 


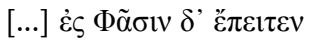

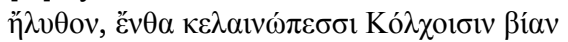

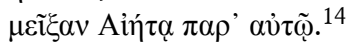

Die Verbindung zwischen Kolchis und Afrika wurde später übrigens von Hanns Jenny Jahnn wieder aufgegriffen, der 1924 Medea als Afrikanerin auftreten lässt. Jahnns Tragödie, 1926 im Staatstheater zu Berlin von Jürgen Fehling aufgeführt, wirkte sich auch auf die Inszenierungsgeschichte von Grillparzers Medea aus. So ließ Agnes Straub 1933, inspiriert von Jahnn, Grillparzers Medea als dunkelhäutige Afrikanerin mit bunt-exotischer Kleidung die Bühne betreten.

Grillparzer selbst analogisiert das mythische Land der Kolcher nicht einfach mit Afrika, sondern verleiht ihm die synkretistischen Züge einer fantastischen Konstruktion. Ein wichtiger Aspekt ist die Kontamination der kolchischen Frauen mit den Amazonen, die gleich zu Beginn der Trilogie deutlich wird:

[...] Medea, Gora, Peritta, Gefolge von Jungfrauen.

Beim Aufziehen des Vorhanges steht Medea im Vorgrunde mit dem

Bogen in der Hand in der Stellung einer, die eben den Pfeil

abgeschossen. An den Stufen des Altars liegt ein, von einem Pfeile

durchbobrtes Reb.

JUNGFRAUEN. die entfernt gestanden, zum Altare bineilend

Das Opfer blutet!

MEDEA. in ibrer vorigen Stellung

Traf's?

EINE DER JUNGFRAUEN.

Gerad' ins Herz!

MEDEA. indem sie den Bogen abgibt

Das deutet Gutes; laßt uns eilen denn!

Geh' eine hin und spreche das Gebet.

GORA. zum Altar tretend

Darimba, mächtige Göttin

Menschenerhalterin, Menschentöterin

Die den Wein du gibst und des Halmes Frucht

Gibst des Weidwerks herzerfreuende Spende

Und des Todfeinds Blut:

Darimba, reine, magdliche

Tochter des Himmels,

Höre mich!

CHOR.

Darimba, mächtige Göttin,

Darimba! Darimba! ${ }^{15}$

14 Pindar: Pythien, IV 212. Übersetzung: ,[...] Zum Phasis kamen sie [die Argonauten] dann, | wo mit schwarzgesichtigen Kolchern Gewalt | sie mischten vor Aietes selbst.'

15 Grillparzer: Der Gastfreund, V. 1-13. 
Das überlieferte Bild Medeas als Zauberin wird hier durch ihre Charakterisierung als wehrhafte Amazone angereichert. Als routinierte Jägerin erlegt sie für eine Opferhandlung ein Reh und führt eine Schar von Jungfrauen an, die nach dem Vorbild der Amazonen von den Männern abgegrenzt ist. So fällt eine ihrer Jungfrauen, Peritta, bei Medea in Ungnade, weil sie sich mit einem Mann eingelassen hat. Und weiblich ist auch die Gottheit, die Medea und ihre Jungfrauen verehren, Darimba, die an Demeter sowie an die nach Ovid auch in Kolchis verehrte Göttin Diana erinnert - deren Priesterin Goethes Iphigenie war -, zugleich aber afrikanisch klingt. Im Unterschied zu Diana repräsentiert Darimba ferner eine vorolympische, matriarchalische Gottheit, die den im Naturzyklus verankerten Wechsel von Entstehen und Vergehen symbolisiert.

Das von Grillparzer geschilderte Kolchis ist allerdings keine matriarchalische Welt. Die Jägerinnen sind eine Enklave, die einen Überrest des Matriarchats in einer patriarchalischen Kultur darstellt. ${ }^{16}$ Noch höher als Darimba rangiert eine männliche Gottheit. In den Entwürfen heißt dieser Gott Brontes beziehungsweise Bronto. ${ }^{17}$ In der endgültigen Fassung trägt der Gott den Namen Peronto. ${ }^{18}$ In der äußeren Erscheinung seines Kultbildes - eine „kolossale Bildsäule eines nackten, bärtigen Mannes [...], der in seiner Rechten eine Keule, um die Schultern ein Widderfell trägt" ${ }^{\text {"19 }}$ - ähnelt der in Kolchis verehrte Gott Peronto Herakles, dem Helden par excellence. Der Gott Peronto, mit einem Widderfell geschmückt, ist in der Tragödie mit dem Goldenen Vlies eng verbunden, welches das verhängnisvolle heroische Streben nach Herrschaft und Heldenruhm symbolisiert. Das Unheil des heroischen Machtstrebens geht bereits aus der hintergründigen Devise des Gottes hervor: „Nimm Sieg und Rache hin“. Nur vordergründig stellt sie einen optimistischen Kampfspruch dar, in Wirklichkeit repräsentiert sie die tragische Dialektik von Triumph und Vergeltung, errungenem Sieg und dafür erlittener Rache - die Ab-

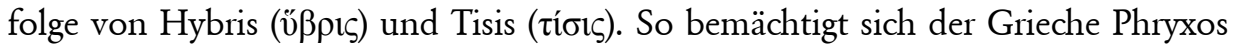
des Vlieses, das er in Delphi der Statue eines Gottes raubt, der Peronto ähnelt, und rechtfertigt seine Tat durch einen angeblichen Traum, in dem der Gott ihm das Vlies anvertraut habe. Für diesen Tempelraub muss Phryxos später in Kolchis büßen, indem er von Aietes im Namen Perontos erschlagen wird. Seinerseits verletzt Aietes, um in den Besitz des Vlieses zu gelangen, das heilige Gastrecht gegenüber dem asylsuchenden Phryxus und verliert später durch Jason seinen Sohn Absyrtos und seine Tochter Medea. Jason schließlich wird in den Besitz des Vlieses gelangen, erntet aber Medeas Rache. Zu Darimba und Peronto gesellt sich noch

16 Gheri: Franz Grillparzer (Anm. 1), 259.

17 Er heißt so nach einem der drei Zyklopen, die laut Hesiods Theogonie Gaia mit Uranos

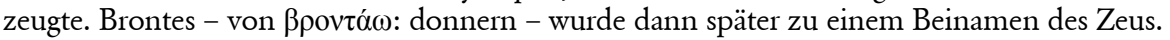

18 Der Name Peronto klingt übrigens an Perinthos an, eine thrakische Siedlung, die von den Griechen kolonisiert und später in Herakleia umbenannt wurde. Er verweist damit proleptisch auf das Schicksal der Kolcher, die im 7. Jahrhundert ebenfalls zur griechischen Kolonie wurden.

19 Grillparzer: Der Gastfreund, Bühnenanweisung zum I. Akt. 
der Unterweltgott Heimdar, der auf den nordischen Gott Heimdallr zurückverweist, nach der Edda Wächter der Götter und Stifter der menschlichen Ständeordnung und bei Grillparzer Gott der Toten. ${ }^{20}$

Den Gegensatz zu den Griechen markiert Grillparzer nicht nur durch die kultische, sondern auch die stilistische Dimension. Die Griechen reden in eleganten Jamben, die Kolcher in holprigen freien Versen. ${ }^{21}$ Auch kontrastiert Grillparzer die überbordende Eloquenz des griechischen Gastes Phryxus, die auch Medeas Missfallen erregt, ${ }^{22}$ mit der Wortkargheit und rhetorischen Unbeholfenheit der Kolcher, deren barbarolexis gelegentlich auch durch Inversion und Ellipse der Hilfsverben bekräftigt wird:

\section{AIETES. (näher tretend mit gedämpfter Stimme) \\ Angekommen Männer \\ Aus fernem Land \\ Bringen Gold, bringen Schätze, \\ Reiche Beute. ${ }^{23}$}

\section{Eine Barbarin in Korinth}

Im Vergleich zu seinen Vorgängern ist Grillparzer sichtlich darum bemüht, Medea zu humanisieren und lässt sie durch eindeutige Sympathielenkung zur menschlichsten Gestalt der Tragödie avancieren. Zu ihr gesellt sich auch die alte Amme Gora als Chorfigur. Grillparzer exkulpiert Medea insofern von ihrer Untat, als er sie als das Resultat einer progressiven Ausgrenzung durch die Griechen erscheinen lässt, die Medea nicht nur erniedrigen und schließlich verstoßen, sondern ihr auch die eigenen Kinder entfremden, so dass sich die Schuldfrage an dem Kindsmord von Medea weg zur griechischen Kultur verschiebt. Medea selbst formuliert die Aitiologie ihrer Missetat in den Versen:

MEDEA. [...] Man hat mich bös genannt, ich war es nicht:

Allein ich fühle, daß man's werden kann. ${ }^{24}$

Grillparzers Trilogie ist insofern ein radikaler Gegenentwurf zu Goethes Iphigenie, ${ }^{25}$ als gerade die Nicht-Griechin Medea jetzt zur Identifikationsfigur avanciert, während das Griechentum sich als eine kolonialistische Kultur entpuppt, die andere Zivilisationen ausgrenzt und unterjocht.

20 „MEDEA. Du hast mir wohl selbst erzählet, | Oft, daß Menschen, die nah dem Sterben, Heimdar sich zeige, der furchtbare Gott, | Der die Toten führt in die schaurige Tiefe.“ (Grillparzer: Die Argonauten, V. 566-570).

21 Grillparzer: Werke in sechs Bänden, Bd. 2 (Anm. 1), 805.

22 „AIETES. Medea! | MEDEA. Vater! | AIETES. Was denkst du? | MEDEA. Ich? nichts! | AIETES. Vom Fremden mein' ich. | MEDEA. Er spricht und spricht; | Mir widert's!“ (Grillparzer: Der Gastfreund, V. 365-367)

23 Ebd., V. 97-100.

24 Grillparzer: Medea, V. 1849-1850.

25 Dazu auch Gheri: Franz Grillparzer (Anm. 1), 258. 
Leitmotivisch ist die Bezeichnung Medeas als Barbarin. Der Terminus ,barbarisch $^{6}$ als Sammelbezeichnung für alle Nicht-Griechen ${ }^{26}$ war anfänglich etwa bei Hesiod nicht unbedingt negativ konnotiert, ${ }^{27}$ nahm aber bald, seit dem 6 . Jahrhundert, die pejorative Bedeutung von ,wild', ,unzivilisiert' und ,kulturell unterlegen' an. In diesem diskreditierenden Sinne wird auch Medea als Barbarin apostrophiert, wiewohl sie das Griechische problemlos versteht und sich wie die Griechen in tadellosen Jamben artikuliert. ${ }^{28}$ Bereits im zweiten Teil bedient sich Jason trotz seiner Liebe zu Medea der Begriffsantithese ,hellenisch' versus ,barbarisch':

Ich ein Hellene, du Barbarenbluts,

Ich frei und offen, du voll Zaubertrug,

Ich Kolchis' Feind, du seines Königs Kind[.. ${ }^{29}$

Im dritten Teil der Trilogie, der Medeas Lage in Korinth schildert und den man als die Tragödie einer misslungenen Integration bezeichnen könnte, wird ihre barbarische Herkunft zum Leitmotiv. Gleich bei ihrer ersten Begegnung mit Kreon, König von Korinth, und seiner Tochter Kreusa stempelt letztere Medeas Namen als „Barbarennamen" ab und schreckt vor ihrem bloßen Anblick zurück. ${ }^{30}$ Kreusa begeht ferner gegen Medeas Status einer Mutter einen Affront, indem sie in ihrer Anwesenheit Medeas Kinder als „Waisen“ bemitleidet und sich ihnen als Ersatzmutter anbietet. ${ }^{31}$ Ihre Ausgrenzung in Kolchis erlebt Medea auch als soziale Deklassierung und Verlust ihrer ehemaligen privilegierten Stellung als Königstochter:

26 Zum Gegensatzpaar Hellenen - Barbaren vgl. Reinhart Koselleck: Vergangene Zukunft. Zur Semantik geschichtlicher Zeiten. Frankfurt am Main 1979, 218-229.

27 In der Einleitung zu den Historien spricht Herodot parallelisierend vom Ruhm der Grie-

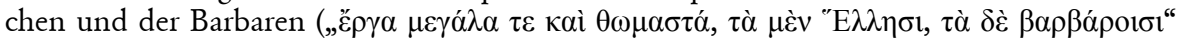
[Herodot: Historien, Bd. 1 (Anm. 12), Einleitung]).

28 Dadurch stößt die Inszenierung kultureller Alterität bei Grillparzer freilich an ihre Grenzen. Offenbar sprengte das im eigentlichen Sinne Barbarische den Rahmen des auf einer klassizistischen Bühne Möglichen und hätte auch Grillparzers Konzept unterminiert, das Barbarische als das Menschliche dem Zuschauer so nah wie möglich zu rücken, wodurch das Plädoyer für das Andere aber letztlich in eine Apologie des Eigenen mündet. Medeas Barbarentum bekundet sich eher symbolisch auf der Ebene der Kostümierung - sie trägt einen roten Schleier auf dem Haupt und verweigert die griechische Frauentracht - und durch ihre Charakterisierung als Zauberin.

29 Grillparzer: Die Argonauten, V. 1204-1206.

30 „KREUSA. [...] | Du warst kaum fort, da scholl’s im ganzen Lande | Von gräßlich wilden Taten, die geschehn, | In Kolchis ließen sie dich Greuel üben, | Zuletzt verbanden sie als Gattin dir | Ein gräßlich Weib, giftmischend, vatermörd'risch. | Wie hieß sie? - Ein Barbarenname war's - | MEDEA. mit ihren Kindern vortretend Medea. | Ich bin's! | KÖNIG. Ist sie's? | JASON. dumpf Sie ist's. | KREUSA. an den Vater gedrängt Entsetzen!“ (Grillparzer: Medea, V. 326-332)

31 „KREUSA. zu den Kindern niederkniend Kommt her zu mir, ihr heimatlosen Waisen, | Wie frühe ruht das Unglück schon auf euch; | So früh und ach, so unverschuldet auch. | Du siehst wie sie - du hast des Vaters Züge. Sie küßt das Kleinere.| Bleibt hier, ich will euch Mutter, Schwester sein! | MEDEA. Was nennst du sie verwaist und klagst darob? | Hier steht ihr Vater, der sie Seine nennt | Und keiner andern Mutter braucht's, so lange | Medea lebt. Zu den Kindern: Hierher zu mir! Hierher!“ (ebd., V. 345-353) 
MEDEA. Weil eine Fremd' ich bin, aus fernem Land

Und unbekannt mit dieses Bodens Bräuchen,

Verachten Sie mich, sehn auf mich herab,

Und eine scheue Wilde bin ich ihnen,

Die Unterste, die Letzte aller Menschen,

Die ich die erste war in meiner Heimat. ${ }^{32}$

Bei Grillparzer sind Assimilationsversuche Medeas durchaus zu konstatieren. Gleich im I. Aufzug des dritten Teils legt sie auf symbolische Weise ihre alte Identität $\mathrm{ab}$, indem sie die Instrumente ihrer Zauberkunst zusammen mit dem Goldenen Vlies begraben lässt:

Erster Aufzug

Vor den Mauern von Korinth [...]. Ein Sklave steht rechts im Vordergrunde in einer Grube, mit der Schaufel grabend und Erde auswerfend, Medea auf der andern Seite, vor ibr eine schwarze, seltsam mit Gold verzierte Kiste, in welche sie mancherlei Gerät wäbrend des Folgenden hineinlegt.

MEDEA. Bist du zu Ende?

SKLAVE.

Gleich, Gebieterin!

$[\ldots]$

MEDEA. Zuerst den Schleier und den Stab der Göttin;

Ich werd' euch nicht mehr brauchen, ruhet hier.

Die Zeit der Nacht, der Zauber ist vorbei

Und was geschieht, ob Schlimmes oder Gutes,

Es muß geschehn am offnen Strahl des Lichts.

Dann dies Gefäß: geheime Flammen birgt's,

Die den verzehren, der's unkundig öffnet;

Dies andere, gefüllt mit gähem Tod,

Hinweg ihr aus des heitern Lebens Nähe!

Noch manches Kraut, manch dunkel-kräftger Stein,

Der ihr entsprangt, der Erde geb' ich euch. ${ }^{33}$

Vor allem die Lyra-Szene zu Beginn des II. Aufzugs repräsentiert einen bewegenden Integrationsversuch Medeas, der an Jasons Roheit scheitert. Unter Kreusas Anleitung bemüht sich die bis dahin nur im Bogenschießen erfahrene Fremde, sich das diffizile Lyraspiel anzueignen. Dieses Akkulturationsbestreben entspricht zugleich ihrem Versuch, Jasons Liebe zurückzugewinnen, da Medea ihm auf der Leier ein Lied vorspielen will, das er als Kind gerne sang. Jason aber, der sie permanent mit dem Klischee der Barbarin konfrontiert, beachtet sie kaum. Seine Aufmerksamkeit gilt nur Kreusa, der er die gemeinsam verbrachte Kinderzeit selbstherrlich in Erinnerung ruft, um sie als neue Partnerin zu gewinnen. Vergeblich versucht Medea, seinen blasierten Redeschwall zu unterbrechen. Als sich dann Jason bequemt, das Lied anzuhören, scheitert die überforderte Medea, was Jason erneut Gelegenheit gibt, ihr ihre barbarische Wildheit höhnisch vorzuhalten:

32 Ebd., V. 400-405.

33 Ebd., V. 1-12. 
Siehst du, ich sagt' es wohl, es geht nun nicht!

An andres Spiel ist ihre Hand gewohnt,

Den Drachen sang sie zaub'risch in den Schlaf.

Und das klang anders als dein reines Lied. ${ }^{34}$

Als die weinende Medea die Leier schließlich fallen lässt und Kreusa Jasons Härte moniert, rechtfertigt er seine Brutalität durch die Berufung auf die Götter, als deren Vollstrecker er sich geriert: „Es ist der Götter Hand, was sie nun fühlt | [...] Greif du nicht in der Götter Richteramt! ${ }^{\lceil 3}$ Jasons Aufforderung, Kreusa möge selbst die Leier nehmen, um ihm vorzuspielen, symbolisiert die neue Liebesbeziehung zwischen beiden, die Medea durch ihre Weigerung zu unterbinden versucht, das Instrument abzugeben. Das Zerbrechen der Lyra, die Jason ihr gewaltsam entreißen will, und die Medea schließlich selbst zerstört, repräsentiert das Misslingen ihrer Integration, aber auch ihre Befreiung aus dem ihr aufgezwungenen Weiblichkeitsmodell, das die Frau zu einer Sängerin des männlichen Heroismus degradiert. ${ }^{36}$ Bezeichnenderweise antwortet auf Kreusas entsetzen Ausruf: „Tot“ Medeas befreiender Aufschrei: „Ich lebe! lebe!“汭

\section{3. „Des Vließes mächt'ger Held“}

Ein wichtiger Aspekt der Kulturkritik, die Grillparzer in seiner Trilogie entfaltet, ist seine Demontage des maskulinen und militärischen Heroismus, den Jason verkörpert. In einer Vorarbeit von 1818 heißt es, dass Jason das Paradigma des Heroischen darstellt: „Jason ein glänzender Held. [...] Erinnere dich immer der griechischen Heroenstatuen und denk' dir ihn nackt blos den Helm auf dem Kopfe und das Schwert in der Hand“. ${ }^{38}$ Als Träger des splendor heroicus und als Vollbringer der „kühnsten Tat, | Die noch geschehn, seit Menschen sind und denken“, ${ }^{39}$ repräsentiert Jason allerdings zugleich die Unwerte des westlichen Militarismus und Imperialismus, die Grillparzer in seiner Trilogie zu kritisieren nicht müde wird. Diese Heroismuskritik schlägt sich vor allem in der Charakterisierung Jasons als eines bramarbasierenden und brachialen Schwadroneurs. Symptomatisch ist, dass sich seine Heroisierung und Divinisierung in den Augen Medeas retrospektiv als eine optische Täuschung der Verliebten erweist:

MEDEA. So stand er da an Kolchis' fremder Küste;

Die Männer stürzten nieder seinem Blick,

Und mit demselben Blick warf er den Brand

In der Unsel'gen Busen, die ihn floh,

34 Ebd., V. 900-904.

35 Ebd., V. 910-912.

36 So auch Gheri: Franz Grillparzer (Anm. 1), 275.

37 Grillparzer: Medea, V. 924-925.

38 Grillparzer: Werke in sechs Bänden, Bd. 2 (Anm. 1), 774.

39 Grillparzer: Medea, V. 430-431. 
Bis, lang verhehlt, die Flamme stieg empor

Und Ruh' und Glück und Frieden prasselnd sanken

Von Rauchesqualm und Feuersglut umhüllt.

So stand er da in Kraft und Schönheit prangend,

Ein Held, ein Gott und lockte, lockte, lockte,

Bis es verlockt, sein Opfer, und vernichtet,

Dann warf er's hin und Niemand hob es auf. ${ }^{40}$

Grillparzers Relativierung von Jasons heroischer Größe zeigt sich auch in der Szene von der Eroberung des Goldenen Vlieses - einer Tat, die von Grillparzer womöglich zum ersten Mal auf die Bühne gebracht wurde. Die Platzierung von Drache und Vlies hinter einer Felswand mit einem großen, verschlossenen Tor, das Jason öffnet, um sofort darauf aufschreiend zurückzufahren, ${ }^{41}$ gibt Grillparzer Gelegenheit zu einer Komisierung des Helden, die auch in Medeas ironischem Kommentar nachklingt:

MEDEA. (wild lachend)

Bebst du? Schauert dir das Gebein?

Hast's ja gewollt, warum gehst du nicht?

Starker, Kühner, Gewaltiger!

Nur gegen mich hast du Mut? ${ }^{42}$

Auch gegenüber der Frau, die er später verstoßen will, zeigt sich Jason als nicht besonders couragiert. Während der Auseinandersetzungen mit Medea versteckt er sich hinter Kreusa. ${ }^{43}$ Auch hat er nicht den Mut, sie in ihrem Haus allein aufzusuchen, sondern verlangt von der Amme, dass sie zwischen ihm und Medea vermittelt. $^{44}$

Erst recht hat der gealterte Jason in Korinth seine heroische Aura weitgehend eingebüßt. Er selbst erzählt Kreusa, dass er längst zu einem Niemand geworden ist, den die Passanten bitten, nicht im Wege zu stehen:

JASON. [...] Ich kam den lauten Markt entlang

Und durch die weiten Gassen eurer Stadt -

Weißt du noch, wie durch sie ich prangend schritt

40 Ebd., V. 617-640 (meine Hervorhebung).

41 „JASON. Laß mich Weib! Mir schallt ein höhrer Ruf!| Gegen die Pforten zugehend: Und bärgest du des Tartarus Entsetzen, | Ich steh' dir! | Er haut mit dem Schwerte gegen die Pforte. Tut euch auf, ihr Pforten! - - Ah!| Die Pforten springen auf und zeigen eine innere schmälere Höble, seltsam beleuchtet. Im Hintergrunde ein Baum. An ibm bängt hellglänzend das goldene Vließ. Um Baum und Vließ windet sich eine ungeheure Schlange, die beim Aufspringen der Pforte ibr in dem Laube verborgenes Haupt hervorstreckt und züngelnd vor sich bin blickt. Jason fährt aufschreiend zurück und kommt wieder in den Vordergrund." (Grillparzer: Die Argonauten, V. 1534-1536)

42 Ebd., V. 1537-1540.

43 „MEDEA. Weißt du? - Komm her zu mir! - Weich mir nicht aus! | Verbirg nicht hinter jene dich vor mir!“ (Grillparzer: Medea, V. 1079-1080)

44 „GORA. auf Jason zeigend Den will sie sprechen | Und hat er Mut dazu, tret' er ins Haus. | JASON. Verwegne geh! mein Haß von Anfang her! | Und sag' ihr, daß sie komme, die dir gleicht. | GORA. O gliche sie mir doch! ihr trotztet nicht! | Doch sie wird's noch erkennen und dann weh euch! | JASON. Ich will sie sprechen! GORA. Geh hinein. JASON. Das nicht! | Sie soll heraus! und du geh hin und sag ihr's!“ (Grillparzer: Medea, V. 1309-1316) 
Als ich, vor jenem Argonautenzug,

Hierher kam, von euch Abschied noch zu nehmen?

[...] Jetzt als ich durch dieselben Straßen ging,

Traf mich kein Aug, kein Gruß, kein Wort.

Nur als ich stand, und rings her um mich sah,

Meint' Einer, es sei schlechte Sitte, so

In Weges Mitte stehn und Andre stören. ${ }^{45}$

Ihren Höhepunkt erreicht die Depotenzierung des Helden Jason durch seine Verbannung durch Kreon, der nach dem Mord an seiner Tochter nicht nur Medea, sondern auch den griechischen Heros als einen Befleckten aus Korinth proskribiert. Ihn ereilt am Ende dasselbe Schicksal wie die Barbarin: Er wird in die Wildnis verstoßen, in die er zuvor Medea verbannt hatte. ${ }^{46}$ Dass der herumirrende Held sodann von einem einfachen Landmann erneut geächtet wird, dessen Auftritt bereits als solcher die Ständeklausel verletzt ${ }^{47}$ transponiert die tragische Handlung auf eine komische Ebene und besiegelt endgültig Jasons Prestigeverlust:

LANDMANN. Wer pocht? - Wer bist du Armer? todesmatt?

JASON. Nur Wasser! Einen Trunk! - Ich bin der Jason!

Des Wunder-Vließes Held! Ein Fürst! Ein König!

Der Argonauten Führer Jason, ich!

LANDMANN. Bist du der Jason? so heb dich von hinnen.

Beflecke nicht mein Haus, da du's betrittst.

Hast meines Königs Tochter du getötet

Nicht fordre Schutz vor seines Volkes Tür.

Ergeht hinein, die Türe schließend. ${ }^{48}$

Die Entwertung des Heroismus, den Jason repräsentiert, geht ebenfalls aus der letzten großen Rede Medeas hervor. In ihr lassen sich auch die Spuren von Grillparzers Auseinandersetzung mit Calderón erkennen, mit dem er sich zwischen 1812 und 1822 intensiv befasst hatte. ${ }^{49}$ Gerade die von Medea formulierte Einsicht in die Hinfälligkeit irdischer Größe und irdischen Glücks erinnert an die Erfahrung des desengaño aus Calderóns Drama: ${ }^{50}$

MEDEA. [...]

Erkennst das Zeichen du, um das du rangst?

Das dir ein Ruhm war und ein Glück dir schien?

Was ist der Erde Glück? - Ein Schatten!

Was ist der Erde Ruhm? - Ein Traum!

Du Armer! Der von Schatten du geträumt! ${ }^{51}$

45 Ebd., V. 795-799, 812-816.

46 Ebd., V. 1051.

47 Winkler: Von Iphigenie zu Medea (Anm. 1), 239.

48 Grillparzer: Medea, V. 2292-2299.

491813 hatte Grillparzer eine Szene aus La vida es sueño übersetzt.

50 Waltraud Branscheid: Grillparzer und Calderón. Düsseldorf, Diss. 1963, 56.

51 Grillparzer: Medea, V. 2364-2368. 
Jasons heroische Taten werden somit vor dem Hintergrund dieses christlichbarocken Vanitas-Diskurses als nichtig präsentiert. ${ }^{52}$

\section{Eine fremde Heldin?}

Besitzt dagegen Grillparzers Medea Züge des Heroischen? Lässt Grillparzers Vanitas-Poetik noch irgendeine Form des Heroischen zu? Zu Beginn erscheint Medea als eine Virago und sie ist für Jason eine unverzichtbare Assistenzfigur, indem sie ihn mehrmals vor dem Tode rettet und den Drachen einschläfert, der über das Vlies wacht. Als Tat-Heldin bleibt sie aber in seinem Schatten.

Ein kontrastiver Vergleich mit Euripides erlaubt es, die Spezifik von Grillparzers Medea und auch ihres eventuellen Heroismus näher zu erfassen. In Euripides' Tragödie erscheint Medeia als eine Heroine im altgriechischen Sinne, und zwar nicht nur wegen ihrer halbgöttlichen Herkunft und ihrer Abstammung von Hekate, die Göttin der Hexerei, Magie und Theurgie -, und Medeia ist aufgrund dieser halbgöttlichen Herkunft unsterblich. Heroisch ist ihr Status vor allem insofern, als sie Jason bei seinem Unternehmen tatkräftig unterstützt hat und mit ihm einen ehelichen Bund freiwillig eingegangen ist, als ebenbürtige Partnerin und nicht als Objekt einer Verhandlung zwischen ihrem Vater und dem künftigen Gatten. Dies verleiht Medeia eine Jason gegenüber gleichrangige Rolle. ${ }^{53}$ Heldenhaft ist auch ihr Ethos, weil sie ihrem verletzten Ehrenkodex den Vorzug gibt und dem Diktat der heroischen Rache folgt. ${ }^{54}$ Darin steht sie in markantem Kontrast zu Jason, der bereits ein verbürgerlichter Held ist, sich vorwiegend der Sprache des Kommerzes bedient ${ }^{55}$ und nach dem Verhaltenskodex des damaligen Athen handelt, indem er eine neue Ehe als Bund zwischen zwei Männern schließt, bei dem die künftige

52 Vor dieser Folie gewinnt auch eine Stelle aus dem zweiten Teil der Trilogie ihren ironischen Hintersinn: „ZWEITER ARGONAUT. [...] Ein Held ist wer das Leben Großem opfert | Wer's für ein Nichts vergeudet ist ein Tor!" (Grillparzer: Die Argonauten, V. 754-755) Dass Kreon Medeas mutterlose Kinder zu „künft'gen Helden“ erziehen will, trägt ebenfalls dazu bei, die Sphäre des Heroischen weiter zu disqualifizieren (Grillparzer: Medea, V. 1913).

53

Dazu Mastronarde: „Medea views herself as a heroic partner in Jason's adventures. She is not a normal citizen-woman, but a princess and a saviour, and she has formed her bond with Jason not as a subordinate in an exchange between her father and her husband, but as an equal. She and Jason exchanged the pledge of right hands and the oaths characteristic of xenoi of equal status, and again Aegeus serves importantly as an outsider who confirms Medea's status among the elite." (Donald J. Mastronarde: General Introduction. In: Euripides: Medea, hg. von Donald J. Mastronarde. Cambridge 2002, 9)

54 „For her sense of outrage over the failure of her partner to abide by the heroic code of mutual exchange and loyal good will, she may be compared to the Achilles of the Iliad and the Ajax of Sophocles' eponymous play. Medea repeatedly refers to honour, dishonour, and the avoidance of being laughed at by her enemies, and unlike Achilles, who for a time rejects the heroic code because he perceives it as flawed, Medea makes her tragic decisions because she gives precedence to her heroic status and to following the dictates of the heroic code of retaliation." (ebd.)

55 Mastronarde: General Introduction (Anm. 53), 36. Vgl. Euripides: Medea (Anm. 9), Z. 454, $461,527,532,535,542,560,566,611-612,615,910,960,963$. 
Braut kein Mitspracherecht besitzt. Dagegen spricht und handelt Medeia wie eine Gestalt aus dem Epos. Ihre Sprache und Bilder stammen aus der männlichen Sphäre, sie sind militärisch und agonal konnotiert. ${ }^{56}$ Und auch ihr Handeln ist heroisch, indem sie Demütigungen durch ihre Feinde nicht duldet ${ }^{57}$ und für Jasons Verrat eine außerordentliche, das menschliche Maß sprengende Rache nimmt, die zugleich ihren von Jason befleckten heroischen Ruhm wiederherstellt. Medeias heroisches Ethos kommt in den Versen 807 bis 810 deutlich zum Ausdruck:

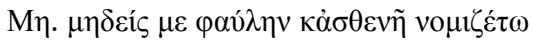

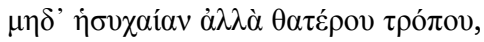

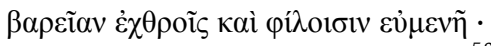

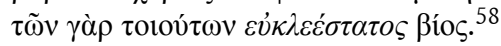

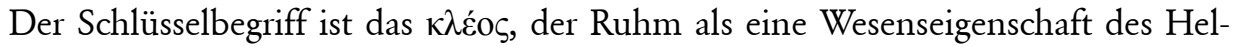
den. Die $\kappa \lambda \varepsilon \dot{\varepsilon} \alpha \dot{\alpha} v \delta \rho \tilde{o} v$ sind die ruhmvollen Taten der Helden, die in den Epen besungen werden. Dadurch assimiliert Medeia eine männliche Kategorie, zu der sich auch Jason bekennt. In einem früheren Passus der Tragödie beobachtet er:

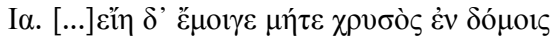

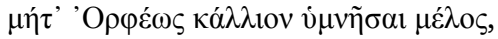

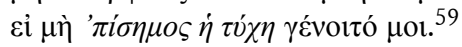

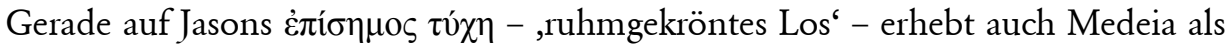
seine ebenbürtige Gattin Anspruch. Sie besitzt das Bewusstsein der eigenen Kraft und die Überzeugung, gerade als Heroine zu einer außergewöhnlichen Rache verpflichtet zu sein. Und auch der Chor unterstützt durch seine Deutung Medeias heroische Selbststilisierung. So singt er im ersten Stasimon:

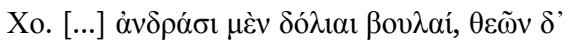

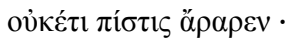

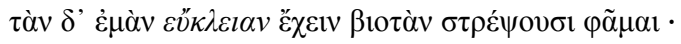

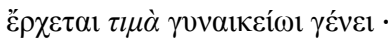

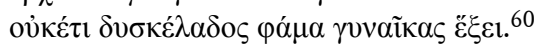

56 Militärisch: Euripides: Medea (Anm. 9), Z. 183, 248-250, 263-264, 390, 394, 403, 408, 466, 597, 765, 852, 938, 1051, 1117, 1185, 1242, 1244, 1246, 1322; athletisch-agonal: Z. 235, 366-367, 546, 585, 1245.

57 Vgl. ebd., Z. 797, 807-810.

58 Ebd., Z. 807-810 (meine Hervorhebung). Übersetzung: ,MEDEIA. Es soll mich keiner | für träge und für schwächlich halten, und auch nicht | für schüchtern, nein, für eine Frau von andrem Schlag: |den Feinden furchtbar, aber wohlgesinnt den Freunden! |Das Leben solcher Menschen erntet höchsten Ruhm.' Diese und die folgenden Übersetzungen von Euripides' Medeia stammen, wenn nicht anders angegeben, von Dietrich Ebener (Euripides: Medeia. In: Euripides: Ausgerwäblte Tragödien, aus dem Griechischen von D. Ebener, hg. von Bernhard Zimmermann. Mannheim 2010, Bd. 1, 136-247).

59 Euripides: Medea, Z. 542-544 (meine Hervorhebung). Übersetzung: ,IASON. [...] Weder mag ich in meinem Hause Gold besitzen, | noch den Orpheus im Gesang von Liedern übertreffen, | Ist mir ein ruhmgekröntes Los nicht vergönnt.‘

60 Euripides: Medea, Z. 412-418 (meine Hervorhebung). Übersetzung: ,CHOR. [...] Die Männer haben listige Pläne, und nicht mehr fest | Steht die bei den Göttern beschworene 
Durch Medeias außerordentliche Rache an ihrem treulosen Mann sollen dem ganzen Frauengeschlecht $\varepsilon \tilde{\kappa} \kappa \lambda \varepsilon i \alpha$ - ,Ruhm', ,Berühmtheit ${ }^{6}$ - und $\tau \imath \mu \eta ́$ - ,Achtung', ,Wertschätzung ${ }^{*}$ - zuteil werden.

Das Problem ist allerdings, dass Medeias Selbstheroisierung die Kategorie des Heroischen ad absurdum führt. ${ }^{61}$ Die Grenzüberschreitung von Medeias Tat, durch welche die Heroine in Bereiche des Verbotenen und Tabuisierten vordringt, verstößt derart massiv gegen das allgemeinmenschliche ethische Empfinden, dass sie die Heldin disqualifiziert und in den Bereich des Pathologischen rückt. Das heroische Übermenschentum, zu dem sich Euripides' Medeia erhebt, ist zugleich ein Unmenschentum. ${ }^{62}$

Auch unter einem anderen Aspekt ist Euripides' Medeia heroisch. Sie nimmt nämlich die Leichen ihrer Kinder auf dem Drachenwagen mit, um sie als Heroen im Heiligtum der Hera Akraia einzusetzen:

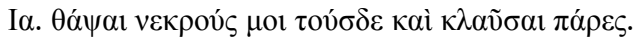

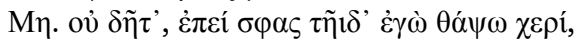

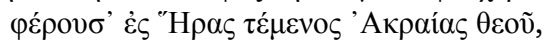

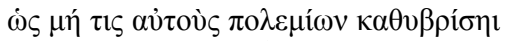

$\tau v \mu \beta \operatorname{ò̀} \varsigma \dot{\alpha} v \alpha \sigma \pi \tilde{\omega} \nu . .^{63}$

Bei Grillparzer spielt dagegen Medeas halbgöttliche Herkunft kaum noch eine Rolle - am Ende entschwebt sie nicht mehr auf dem Drachenwagen, sondern irrt wie eine Heimatlose umher. Sie stiftet auch keinen heroischen Kult ihrer Kinder, die sie jetzt vielmehr als Verräter betrachtet. Von Kreusa manipuliert, haben ihre Kinder sie nämlich verleugnet und ihr die Tochter des Königs als Ersatzmutter vorgezogen haben. $\mathrm{Zu}$ all den Motivationen, die in Medeas Kindermord eine Rolle

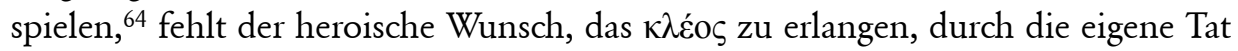

Treue. | Mein Leben aber soll wandeln der Ruhm, | auf dass es Ehren gewinne. | Auszeichnung wird dem Frauengeschlechte zuteil; | misstönend Gerede soll nicht mehr treffen die Frauen.'

61 „Euripides' presentation of the heroic ethos in such a figure (a woman and a mother) and such a deed (the killing of the children) calls the heroic itself into question" (Charles Segal: Euripides' Medea: Vengeance, Reversal and Closure. In: Pallas 45, 1996, 15-44, hier 18). Vgl. auch Winkler: Von Iphigenie zu Medea (Anm. 1), 35, Anm. 49.

62 Wolf Hartmut Friedrich: Vorbild und Neugestaltung. Sechs Kapitel zur Geschichte der Tragödie. Göttingen 1967, 52.

63 Euripides: Medea, Z. 1377-1381. Übersetzung: , JASON. Lass mich die Toten doch bestatten und beweinen! |MEDEA. Niemals! Ich möchte sie mit meiner Hand begraben, | sie tragen in das Heiligtum der Göttin Hera | Akraia, dass kein Feind sie schände und ihr Grab | aufwühle.

64 In Grillparzers Arbeitsnotizen werden die unterschiedlichen Beweggründe für Medeas Tat wie folgt ausdifferenziert: „Medeas Gefühl gegen ihre Kinder muß gemischt seyn aus Haß gegen den Vater, Jason, von dem sie weiß, daß er die Kinder liebt und ihr Tod ihm schmerzlich seyn wird; aus Grimm gegen die Kinder, die sie flohen und ihren Feinden den schmerzlichsten Triumph über sie verschafften; aus Liebe gegen eben diese Kinder, die sie nicht mutterlos unter Fremden zurücklassen will; aus Stolz, ihre Kinder nicht in der Gewalt ihrer Feinde zu lassen." (Grillparzer: Werke in sechs Bänden, Bd. 2 (Anm. 1), 784-785) 
einen unsterblichen Ruhm zu stiften. Heroisch gefärbt sind dagegen auch bei Grillparzers Medea die Wiederbesinnung auf die eigene aristokratische Herkunft ${ }^{65}$ und die Sorge, von ihren Feinden verspottet zu werden, die ein Leitmotiv in Medeas Reden darstellt. ${ }^{66}$ Dadurch knüpft Grillparzer erkennbar an Euripides' Medeia an, die ebenfalls den Gedanken, ihren Gegnern zum Spott zu gereichen, nicht ertragen kann. ${ }^{67}$ Schließlich beabsichtigt Grillparzers Medea, nicht nur die durch Jason erlittene Schmach zu rächen, sondern auch ihren Vater, ihren Bruder sowie schließlich die eigene Kultur selbst zu revanchieren. ${ }^{68}$

In Bezug auf Medeas Heroismus kehrt Grillparzer aber letztlich Euripides' Modell geradezu um. Wirklich heroisch ist bei ihm nicht Medeas Rache, sondern ihre Bereitschaft, dafür zu büßen. Man könnte von einem Heroismus der Buße sprechen, da sich Medea nach ihrer grausamen Tat entscheidet, das Vlies nach Delphi zurückzubringen und sich den dortigen Priestern als Opfer, darzustellen':

MEDEA. Nach Delphi geh' ich. An des Gottes Altar

Von wo das Vließ einst Phryxus weggenommen

Häng' ich, dem dunkeln Gott das Seine gebend,

Es auf, das selbst die Flamme nicht verletzt

Und das hervorging ganz und unversehrt

Aus der Korintherfürstin blut'gem Brande;

Dort stell' ich mich den Priestern dar, sie fragend,

$\mathrm{Ob}$ sie mein Haupt zum Opfer nehmen an,

$\mathrm{Ob}$ sie mich senden in die ferne Wüste

In längerm Leben findend längre Qual. ${ }^{69}$

Zwar wird Medeas Haltung von Grillparzer nicht ausdrücklich als heroisch bezeichnet, sie erfüllt jedoch zwei Kriterien des Heroischen: das Wagnis des eigenen Lebens und die große, glorreiche Tat. Indem sich Medea freiwillig nach Delphi begibt, wagt sie ihr Leben und vollendet auch erst Jasons große Tat, indem sie das

65 Diese Besinnung erfolgt auch ex negativo durch Gora, die Medea an den Verlust ihres aristokratischen Status und ihrer Würde erinnert: „GORA. vortretend Sie meinen, du würdest gehn, | Den Haß bezähmend und die Rache. | Die Törichten! | Oder wirst du es? Wirst du's? | Fast glaub' ich, du tust's, | Denn nicht Medea bist du mehr, | Des Kolcherkönigs königlicher Sproß, | Der erfahrnen Mutter, erfahrnere Tochter; | Hättest du sonst geduldet, getragen | So lange, bis jetzt?“" (Grillparzer: Medea, V. 1158-1168)

So fühlt sich Medea ständig von Kreusa und Jason verspottet: „MEDEA. [...] Lachst du? Du sollst noch weinen, sag' ich dir! | KREUSA. O strafen mich die Götter, lacht' ich jetzt!“ (ebd., V. 1645-1646); „MEDEA. sich halb aufrichtend und nur mit den Knien auf den Stufen liegend So kniet' ich, so lag ich, | So streckt' ich die Hände aus, | Aus nach den Kindern und bat | Und flehte: Eines nur, | Ein einziges von meinen Kindern - | Gestorben wär' ich, mußt' ich das Zweite missen! - | Aber auch das eine nicht! - Keines kam. | Flüchtend bargen sie sich im Schoß der Feindin | Aufspringend: Er aber lachte drob und sie!" (ebd., V. 1731-1739); „MEDEA: [...] Sie aber freun sich hier und lachen mein!“‘ (ebd., V. 2137)

Euripides: Medea, Z. 383, 404, 797, 1049-1050, 1355, 1362.

68 Dies geht aus den Worten Goras hervor: „GORA. So straf' ihn, triff ihn, | Räche den Vater, den Bruder, | Unser Vaterland, unsre Götter, | Unsre Schmach, mich, dich!“ (Grillparzer: Medea, V. 1221-1224) 
Goldene Vlies nach Delphi zurückbringt. Es handelt sich aber im Unterschied zu Jason um einen ausgeprägt ethisierten Heroismus, der vom Gedanken der Sühne bestimmt wird. Es ist ein christlich-humanistisches Heroismusmodell, das sich vom rassistisch grundierten militärischen Heroismus Jasons absetzt und das Medea nicht zufällig nach Delphi als Wiege der Humanitätsidee führt.

Am Schluss der Trilogie kontrastiert Grillparzer die Würde von Medeas Haltung, ihre heroische Bereitschaft zur Selbstüberwindung mit Jasons denkbar unheroischer Verzweiflung:

JASON. Verwaist! Allein! O meine Kinder!

MEDEA.

Trage!

JASON. Verloren!

MEDEA. Dulde!

JASON. Könnt' ich sterben!

MEDEA.

Büße!

Ich geh' und niemals sieht dein Aug' mich wieder! ${ }^{10}$

Auch in der letzten Stichomythie steht somit der christliche Begriff der Buße, der $\mu \varepsilon \tau$ óvora, im Vordergrund.

Dadurch verliert die fremde Heldin ihre Fremdheit. Trotz ihres betonten Exotismus wird sie zum Sprachrohr und Vehikel von Grillparzers humanistischchristlicher Ethik. Die kolchische Zauberin wird abendländisch überformt. Dies bedeutet andererseits aber auch, dass das Humanitätsideal, zu dem sich Grillparzer bekannte, im imperialistischen Europa inzwischen offenkundig so fremd geworden war, dass es nur von einer fremden Heldin verkörpert werden konnte.

70 Ebd., V. 2373-2375. 
